\title{
2220. Experimental research and numerical simulation of influence of pre-stress values on the natural vibration frequency of concrete simply supported beams
}

\author{
Jie Li ${ }^{1}$, Feng Zhang ${ }^{2}$ \\ ${ }^{1}$ Nanjing University of Aeronautics and Astronautics, Nanjing, 210016, China \\ ${ }^{2}$ Xin Jiang Vocational and Technical College of Communications, Wulumuqi, 831401, China \\ ${ }^{1}$ Corresponding author \\ E-mail: ${ }^{1} l j o k \_007 @ 126 . c o m,{ }^{2} 2849840669 @ q q . c o m$
}

Received 22 May 2016; received in revised form 5 August 2016; accepted 8 August 2016

DOI https://doi.org/10.21595/jve.2016.17196

\begin{abstract}
To study the relationship between the fundamental frequencies and pre-stress values, based on experiments and numerical simulation, the simply supported was excited under different pre-stress values through using the hammering method. The collected acceleration signals in the mid-span of the beam were processed and analyzed to obtain the relationship between the fundamental frequency and pre-stress values. As shown from the analyzed result, the relationship between the fundamental frequency and pre-stress values could not be explained better based on the traditional "Euler-Bernoulli Beam Equation" theory. The fundamental frequency of the pre-stressed beam with the hammering method would be increased with the increasing pre-stress value. When the hammering method was introduced into the numerical simulation, its analysis result and the experimental result were very approximate and presented a small error, which indicated the feasibility and accuracy of the analyzed method. When the pre-stress value was a constant, the fundamental frequency of the beam would be increased with the increasing eccentric distance of the pre-stressing tendon. When other conditions were constant, the fundamental frequency of the beam presented a decreasing trend with the counterweight at the upper part of the beam.
\end{abstract}

Keywords: pre-stress beam, concrete beam, fundamental frequency, pre-stress value, hammering method.

\section{Introduction}

Pre-stress bridges are widely applied in the world. For bridges which are being used now, bridge structure will age with the increasing service life of bridges. In particular, effective pre-stress of the pre-stress bridge will suffer from losses. The actual effective pre-stress of bridges usually deviates from the design value for the reason of construction deviations. Effective pre-stress is a key factor for the safety of these kinds of bridges. Therefore, how to recognize the effective pre-stress of the bridge is a very important research direction. The stress state and bearing capacity of the bridge can be reflected through the dynamic characteristic of the bridge structure, and therefore the stiffness change of the structure will be caused by the loss of pre-stress, resulting in the simultaneous change of both structure and natural frequency of the bridge. Therefore, detection methods based on pre-stress loss regarding the natural frequency were researched by many scholars [1-3].

The change of pre-stress values will influence the dynamic characteristic of the bridge. Particularly, the natural vibration frequency of the bridge is sensitive to pre-stress values to some degree. According to some experimental studies, the natural frequency of the pre-stress beam has a certain mathematical relationship with pre-stress values. Namely, the natural vibration frequency of the beam is increased with the increasing pre-stress values, which provides a theoretical basis for detecting the pre-stress loss of the bridge based on the natural frequency. Therefore, recognizing the effective pre-stress of the bridge through analyzing the change of dynamic parameters of the bridge is a feasible method.

At present, "Euler-Bernoulli Beam Equation" is the most mature theory for computing the 
beam fundamental frequency. However, after introducing pre-stress to the beam, the theory generally deems pre-stress as axial pressure of the beam. In other words, as explained on the basis of Euler-Bernoulli theory, the pre-stress beam fundamental frequency will decreased with the increase of pre-stress value [4-6]. This effect is called as "compression softening". K. Dai [7], A. Dall'Asta [8] and A. Kerr [9] thought that the fundamental frequency of a pre-stress concrete structure will not be influenced by pre-stress value, namely fundamental frequency and pre-stress value are mutually independent. Based on a nonlinear motion model, A. Kerr [10] derived a vibration equation, where a vibration beam was independent from fundamental frequency. Meanwhile, experimental researches of many scholars also showed that the pre-stress beam fundamental frequency will be increased with the increase of pre-stress value, which is completely contrary to the traditional theories of mechanics. Aiming at this problem, a lot of scholars proposed new theories and methods to explain this problem. For example, Saiidi and Zhang proposed rigidity modification formulas and frequency computation formulas for pre-stress beams based on a lot of experimental data $[11,12]$. However, above formulas are still based on empirical formulas which are obtained by some experiments, so they are restrained in theory and universality.

In conclusion, current researches on relations between pre-stress beam and fundamental frequency have a lot of disputes, while quantitative relations and mature theoretical explanations between them have not been formed. Most of current researches are based on derivation of independent theories and formulas or based on finite element model and innovation of analysis methods. Methods with combination of experimental model and finite element method still require deeper exploration. There are a lot of methods for specimen excitation $[13,14]$, where the hammer excitation method is simple, rapid and relatively accurate, so that the paper adopted the method of hammer for excitation.

The paper intended to take a concrete simply supported beam with pre-stress rebar eccentrically arranged on the bottom as the analyzed model. By analyzing fundamental frequencies of the beam, which were measured by the hammering method under different pre-stress values, the paper quantitatively analyzed and obtained relations between beam fundamental frequency and stress value. The paper also applied the hammering method in finite element analysis. Through the same method, explanations of traditional theories towards the problem could be avoided, and the accurate relations between beam fundamental frequency and pre-stress value could be obtained. Based on this model, the paper further analyzed and obtained relations between beam fundamental frequency, pre-stress value, eccentric distance and balance weight.

\section{Analytical method of traditional theories}

At present, analysis on the pre-stress concrete beam mostly considers pre-stress within the beam as the external force of the beam. Based on the analytical theory, the system of the pre-stress simply supported beam can be simplified as follows. The simplified diagram is as shown in Fig. 1.

The beam was considered as an isotropic material when a theoretical analysis on the pre-stress concrete beam was conducted based on traditional mechanics theories. Namely, the vibration equation of the supply supported beam could be obtained through following plane cross-section assumption in the process of analysis:

$E I \frac{\partial^{4} y(x, t)}{\partial x^{4}}+T \frac{\partial^{2} y(x, t)}{\partial x^{2}}+\bar{m} \frac{\partial^{2} y(x, t)}{\partial x^{2}}=0$,

wherein, $E I$ is the flexural stiffness of the beam; $T$ indicates the equivalent concentrated force of the pre-stressing tendon; $\bar{m}$ means the partial mass of the simply supported beam. Through solving the above formula, the calculation formula of natural vibration frequency of the simplified pre-stressed simply supported beam was obtained as follows [15-17]: 
$\omega_{n}=\sqrt{\left(\frac{n \pi}{l}\right)^{4} \frac{E I}{\bar{m}}-\left(\frac{n \pi}{l}\right)^{2} \frac{T}{\bar{m}}}$

wherein, $n$ is a positive integer; $l$ means the net span of the beam.

As shown from the above formula, the natural vibration frequency of the simply supported beam would be decreased with the increase of $T$. In other words, the natural vibration frequency of the pre-stress concrete simply supported beam would show a decreasing trend with the increasing pre-stress value of the pre-stress steel strand. It is an explanation for the relationship between pre-stress value and fundamental frequency in the pre-stress beam based on classical mechanics theories. However, the experimental result of many scholars is just the opposite. Namely, the fundamental frequency of the pre-stress beam will be increased with the increase of pre-stress values. Based on the experimental phenomena, it is necessary to look for new methods to solve this problem. A lot of scholars have proposed new theories and methods to explain this problem. Saiidi and Zhang proposed their own stiffness-modification formulas and frequency computation formulas of the pre-stress beam based on a lot of experimental data.

The relationship between the fundamental frequency of the pre-stress beam and pre-stress value cannot be better explained based on classical mechanics theories. Therefore, this paper will conduct a detailed analysis on the problem through adopting experiments.

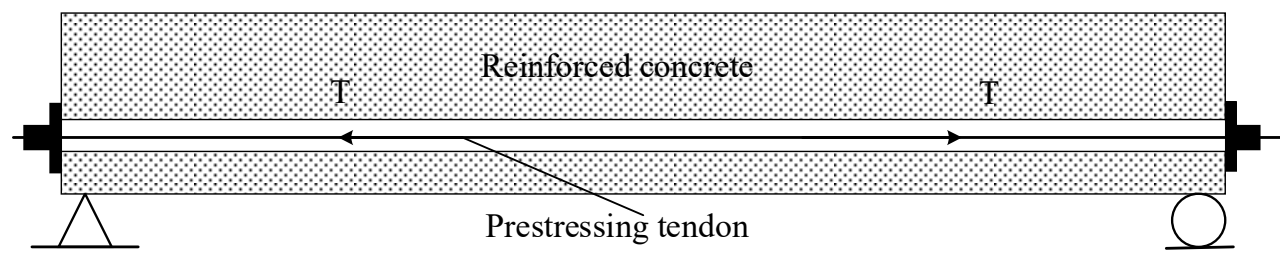

a) Schematic diagram for the actual stress of the pre-stress concrete simply supported beam

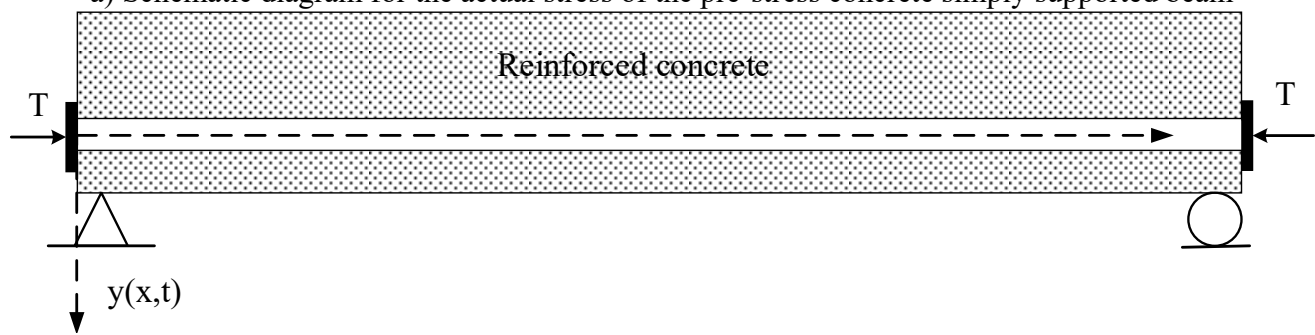

b) Schematic diagram for the simplified stress of the pre-stress concrete simply supported beam

Fig. 1. A simplified analysis on the dynamic of the pre-stress concrete simply supported beam

\section{Experimental proposal for the model of the pre-stress simply supported beam}

The rectangular section was applied to the experimental model of the pre-stress concrete simply supported beam. The test piece was $3.2 \mathrm{~m}$ long. The beam was $0.36 \mathrm{~m}$ high and $0.25 \mathrm{~m}$ wide. The strength grade of concrete was C40. Longitudinal steel adopted HRB400E grade ribbed steel with the diameter of $14 \mathrm{~mm}$. Stirrup adopted HPB400 grade steel with the diameter of $6 \mathrm{~mm}$. The protective layer was $c=20 \mathrm{~mm}$ thick. 1X7-15.2-1860 pre-stress steel strand was applied. Elasticity modulus was $E p=1.95 \times 10^{5} \mathrm{MPa}$. A pre-stress pipe was pre-buried at the bottom of the beam. The internal diameter of the pipe was $2 \mathrm{~cm}$. At the suitable period after completing casting, post-tensioning method was adopted to add pre-stress into the beam. To prevent the stress concentration of the end, a steel plate of $100 \mathrm{~mm} \times 100 \mathrm{~mm} \times 5 \mathrm{~mm}$ was buried here. The model dimension of the rectangular beam was show in the following.

To simulate the relationship between different pre-stress values and the fundamental frequency of the beam, pre-stress within the beam was applied and signals were collected by steps. Centre 
hole jack was applied to extrude the single steel strand of various beams. Digital-display oil pressure gauge was used to control the tension force. An acceleration sensor was placed in the mid-span of the beam. After loading each level of pre-stress values, loading equipment was removed from the beam end and the hammering method was adopted to collect the acceleration signals of the beam. Adopting different test and analytical methods would have a great influence on the experimental result. There might be a big error or a wrong result if the most suitable method was not adopted. Especially, a large number of methods could excite the structure. As a result, it was necessary to carefully select an excited method.

In summary, the dynamic characteristic of the pre-stress simply supported beam under different pre-stress values was mainly studied here. Through combining with the above experimental methods and processes, the experimental proposal shown in Table 1.

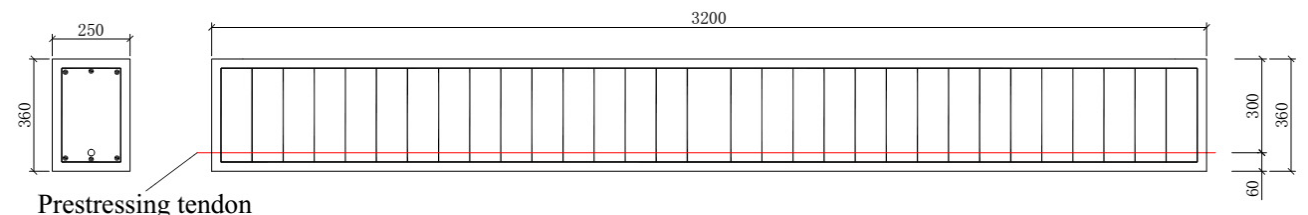

Fig. 2. Dimension of the pre-stress concrete beam (mm)

Table 1. Operating conditions of test

\begin{tabular}{|c|c|c|c|c|c|}
\hline Condition & Condition 1 & Condition 2 & Condition 3 & Condition 4 & Condition 5 \\
\hline $\begin{array}{c}\text { Pre-stress } \\
\text { value/kN }\end{array}$ & 0 & 50 & 100 & 150 & 200 \\
\hline Test method & $\begin{array}{c}\text { Hammering } \\
\text { method }\end{array}$ & $\begin{array}{c}\text { Hammering } \\
\text { method }\end{array}$ & $\begin{array}{c}\text { Hammering } \\
\text { method }\end{array}$ & $\begin{array}{c}\text { Hammering } \\
\text { method }\end{array}$ & $\begin{array}{c}\text { Hammering } \\
\text { method }\end{array}$ \\
\hline
\end{tabular}

Loading and hammer tests were conducted according to various operating conditions of the experimental proposal. The acceleration signals of the beam under different levels of pre-stress loads were collected through the acceleration sensor in the mid-span of the beam to conduct a dynamic analysis on the beam. CF092 magneto-electric acceleration sensor was applied in this paper. The sensor was equipped with an extremely low-frequency response and high sensitivity, which showed remarkable effect in safety test of the bridge and earth pulsating test and required passive servo and no power supply. The test field shown in Fig. 3.

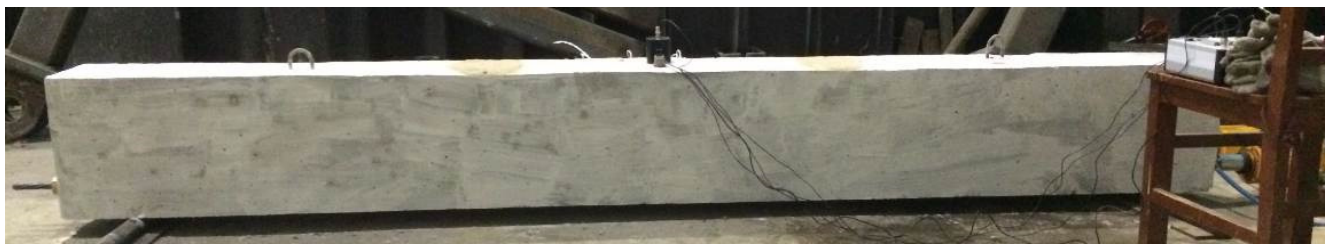

Fig. 3. The experimental process of the pre-stressed concrete beam

\section{Result analysis of model experiments}

According to the experimental proposal, the hammering method was adopted at $1 / 3$ place of the simply supported beam. The acceleration signals of the beam were collected through the magneto-electric acceleration sensor which was placed in the mid-span of the beam. The hammering method was used again under each operating condition when the vibration signal of the beam was attenuated basically. The hammering method was adopted for three times under each condition to respectively obtain the signal in the mid-span of the beam. Fast Fourier Transform (FFT) algorithm was applied to the collected acceleration signals to obtain the fundamental frequency information of the pre-stress beam, which shown in Fig. 4. 

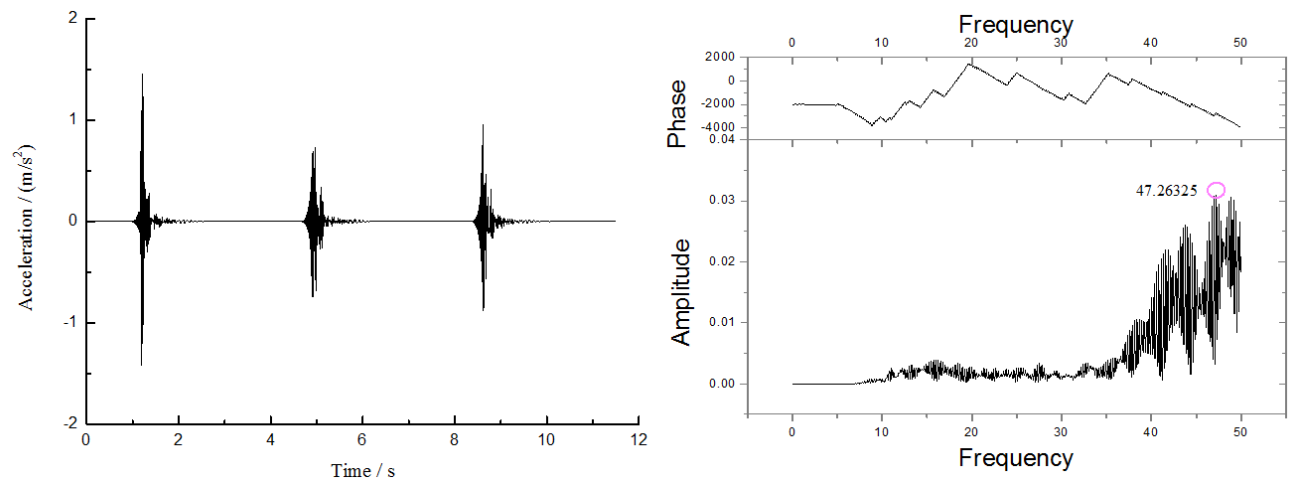

a) $T=0 \mathrm{kN}$
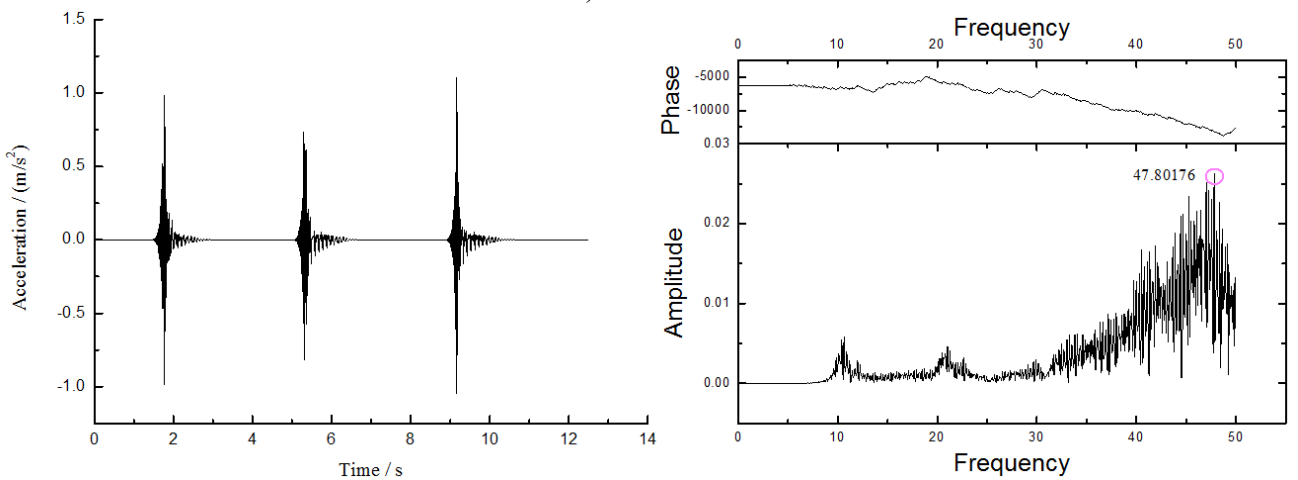

b) $T=50 \mathrm{kN}$
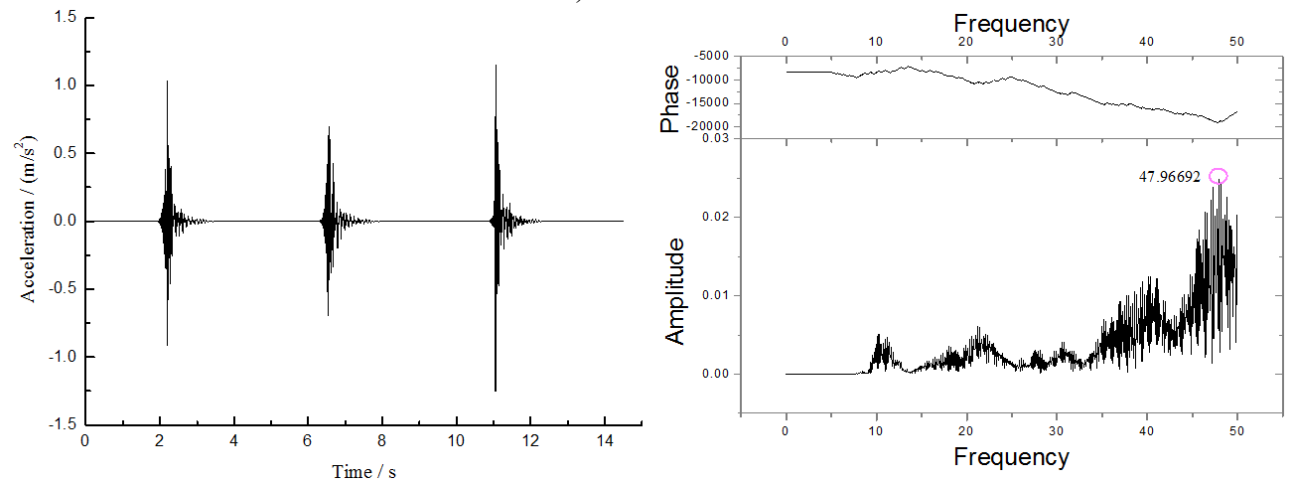

c) $T=100 \mathrm{kN}$
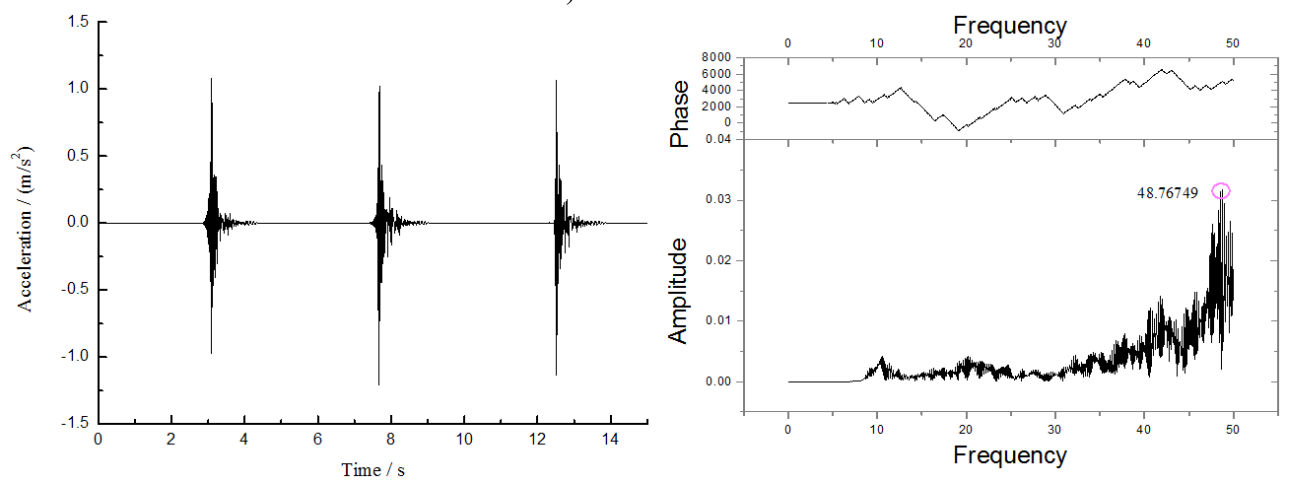

d) $T=150 \mathrm{kN}$ 

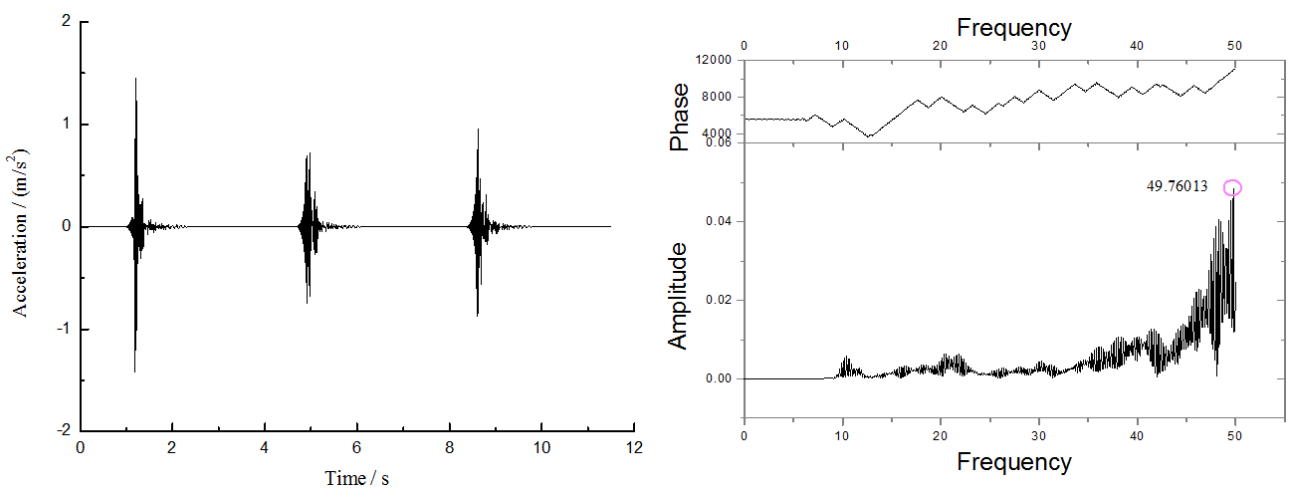

e) $T=200 \mathrm{kN}$

Fig. 4. Time history curve of mid-span acceleration under different pre-stress values

As shown from the collected acceleration signals in the above figure, the acceleration signals of the beam have dramatic changes when instantaneous hammering was applied to the beam through using the rubber hammer. The acceleration signals of the beam gradually tended to be stable after dramatically changing for $1 \mathrm{~s}$. To accurately analyze the dynamic characteristic of the beam and eliminate the influence of various accidental errors in the experimental process, the hammering method was adopted for three times under each condition in the experimental process and the hammering method was used again each time when the last excitation effect became basically stable. Then, FFT method was adopted to analyze collected signals one by one. Collected time-domain signals could be transformed into frequency domain signals to obtain the fundamental frequency information of the beam conveniently. The result of various loading conditions after adopting FFT method was shown in the right side of the above figure. The upper part was phase spectrum and the lower part was amplitude spectrum through which the fundamental frequency value of the beam under various pre-stress values could be obtained.

As shown from the analyzed result of the above figure, the fundamental frequencies of the beam were $47.26325 \mathrm{~Hz}, 47.80176 \mathrm{~Hz}, 47.96692 \mathrm{~Hz}, 48.76749 \mathrm{~Hz}$ and $49.76013 \mathrm{~Hz}$ when the pre-stress values of the beam were $0 \mathrm{kN}, 50 \mathrm{kN}, 100 \mathrm{kN}, 150 \mathrm{kN}$ and $200 \mathrm{kN}$. The fundamental frequency of the beam saw regular changes with the increase of pre-stress values. Namely, the fundamental frequency of the beam reflected an increase trend with the increase of pre-stress values. The experimental phenomenon was contrary to "compression and softening" effect based on "Euler-Bernoulli Equation", but it was consistent with the experimental result of many scholars, which further indicated that existing theories were not appropriate to explain this problem. Therefore, more in-depth studies on the problem should be conducted.

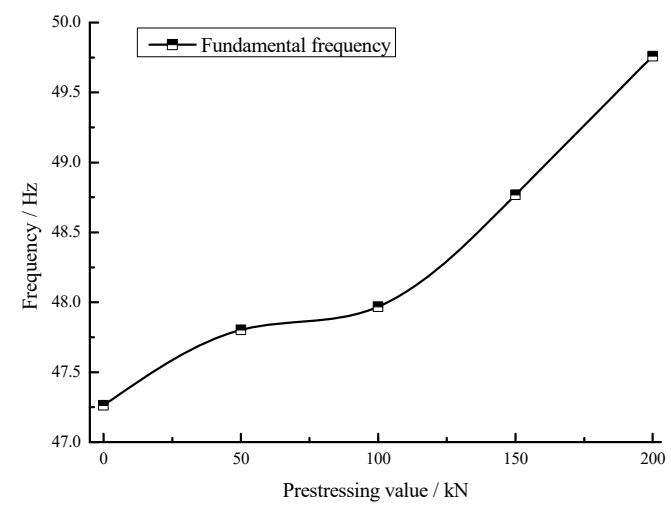

Fig. 5. Curve of relationship between pre-stress and fundamental frequency 
To express the change rule of the fundamental frequency of the pre-stress concrete simply supported beam with pre-stress value more clearly, the analyzed results were put together to conduct a comparative analysis and obtain a variation curve in Fig. 5.

As shown in Fig. 5, the fundamental frequency of the pre-stress simply supported beam showed an increasing trend with the increase of pre-stress values, and the fundamental frequency of the beam belonged to the first-level growth according to the change of pre-stress value from $0 \mathrm{kN}$ to $50 \mathrm{kN}$. The fundamental frequency of the beam would increase to the final $200 \mathrm{kN}$. The increase amplitudes for the fundamental frequency of the beam were $1.1 \%, 1.5 \%, 3.2 \%$ and $5.3 \%$ respectively, showing a gradually increasing trend. The fundamental frequency of the beam was increased with the increase of pre-stress value, but the increase amplitude presented certain fluctuation in the middle. In other words, the increase amplitude did not show linear growth in the middle, but nonlinear growth of certain fluctuation.

\section{Numerical simulation analysis}

\subsection{Finite element model of the simply supported beam}

ABAQUS software was applied to conduct a modeling analysis on the pre-stress concrete simply supported beam. C40 concrete adopted Solide element modeling. Element type was C3D8R. The sectional dimension and material property of concrete were the same with those of the experimental model. Steel strand, main steel bar and stirrup adopted Truss element modeling and element type was T3D2. The material property and sectional dimension of reinforced bars were the same with the model dimension of the experiment. The actual model was tested under different pre-stress value, where the experiments adopted unbounded pre-stress post-tensioning method. In order to make the numerical model more similar with the actual specimen, a pre-stress duct was reserved at a corresponding position of the beam in the numerical model. The pre-stress steel strand passed through the reserved duct from the center position but did not touch the duct wall. The pre-stress steel strand was firmly connected with an anchor head on the beam end, so that a numerical model of the unbounded pre-stress concrete beam was formed. In this way, actual force situations of the specimen can be simulated more truly. Different pre-stress values were adjusted in the experiment, where the pre-stress value of pre-stress steel strand could be adjusted through Keywords (*Initial conditions, type $=$ Stress) in the ABAQUS model after computation of each working condition. The model had 5355 elements, whose displacement and rotation were constrained accordingly at the positions corresponding to the supports of the beam. The whole finite element model of the structure was shown in Fig. 6.

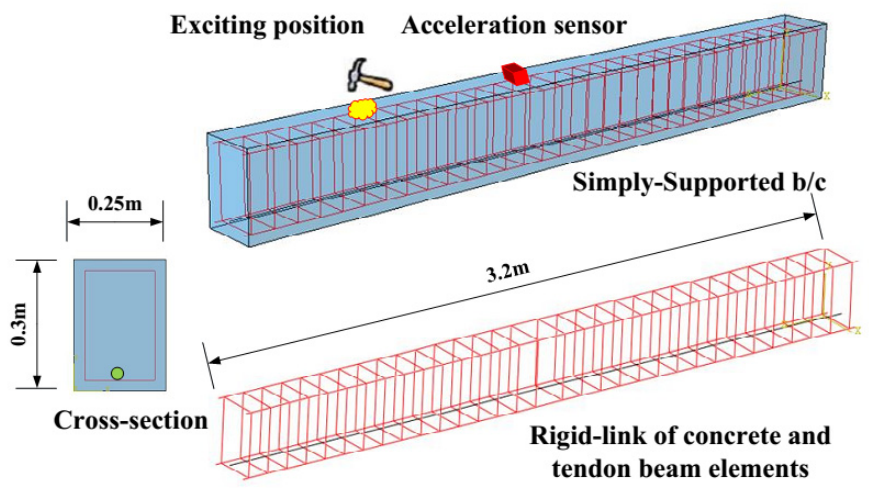

Fig. 6. Finite element model

Finite element software was still applied to analyze structural modal based on the stiffness matrix of structures. From the previous analysis, it could be seen that it was not appropriate to 
solve this problem with traditional mechanics theory. To study the relationship between the fundamental frequency of the pre-stress concreted simply supported beam and pre-stress values based on finite element method, the method adopted in the practical experiment was used to conduct a loading analysis on the above finite element model. Namely, FFT algorithm was used to obtain the fundamental frequency information of the structure through adopting the hammering method to the simply supported beam model and extracting acceleration response signals in the mid-span of the simply supported beam. In the process of the practical experiment, the hammering force value for each time was different to some degree. To simulate the change of force values in the process of the numerical simulation, three hammering values were generated through using the random method and instantaneous loads were applied to the model at corresponding moments. The acceleration signals in the mid-span of the beam of the finite element model under the pre-stress condition of $0 \mathrm{kN}$ and $200 \mathrm{kN}$ were taken as an example. It was shown in Fig. 7.

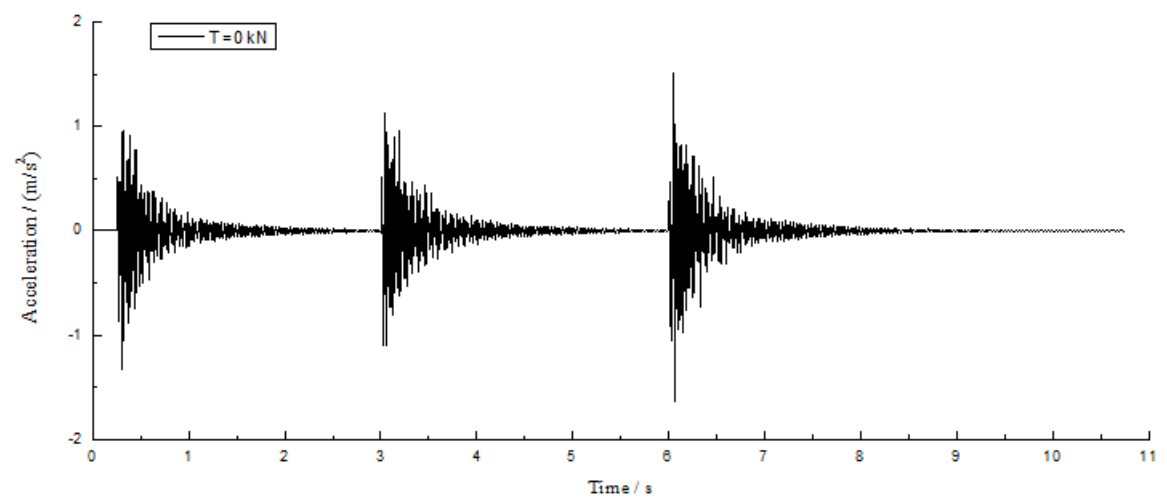

a) Time history curve of $T=0 \mathrm{kN}$ acceleration

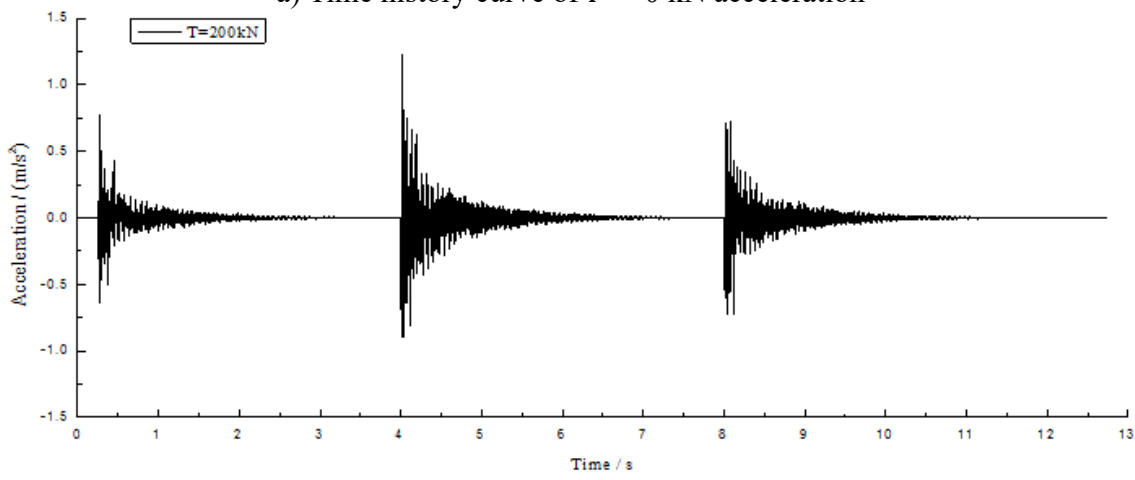

b) Time history curve of $T=200 \mathrm{kN}$ acceleration

Fig. 7. Time history curve of midspan acceleration

In consideration of the energy of exciting force and signal-to-noise ratio of corresponding signals, it was better to select the scope within the frequency band of half-power bandwidth of excitation signals as the credible scope of research. However, the scope could be exceeded if the response signals of the structure were very good. Due to the low frequency of civil engineering structure, rubber hammer heads were generally used in civil engineering experiments because rubber hammerheads could excite low-frequency signals easily because of their narrow frequency band range. The effective band of common rubber heads changed from $0 \mathrm{~Hz}$ to $300 \mathrm{~Hz}$. In the hammer test, frequency spectrum changed gently, whose effect was similar to that of white noises. In addition, signals could be measured in general hammer tests. Therefore, it was relatively reliable to conduct a test analysis through this method. 


\subsection{A study on the relationship between pre-stress and the fundamental frequency of the beam}

The same method was adopted to analyze the collected acceleration signals, obtain the fundamental frequency of the pre-stress simply supported beam under various prestress values based on the finite element method and compare it with the experimental value to obtain in Fig. 8.

As shown in Fig. 8, the errors of the fundamental frequency of the beam obtained by the experimental model and finite element model were within $1 \%$ and the fundamental frequency of the beam had the same change rule with pre-stress value. Namely, fundamental frequency was increased with the increase of pre-stress value. When pre-stress value increased from $0 \mathrm{kN}$ to $200 \mathrm{kN}$, the fundamental frequency of the experimental model was increased by $5.28 \%$ while the fundamental frequency of finite element model was increased by $5.06 \%$. Under other various levels of pre-stress loads, the fundamental frequencies of experimental model and numerical model showed the same order of growth trend. According to the above analysis, the real dynamic signals of the beam could be collected better to obtain the relationship between the accurate fundamental frequency of the pre-stress beam and pre-stress values when the hammering method was introduced into the numerical model.

To quantitatively compare and analyze the difference between the experimental method and digital-analog method in the problem, the fundamental frequency information of the beam under above loading conditions was compared to obtain a comparison curve shown in Fig. 9.

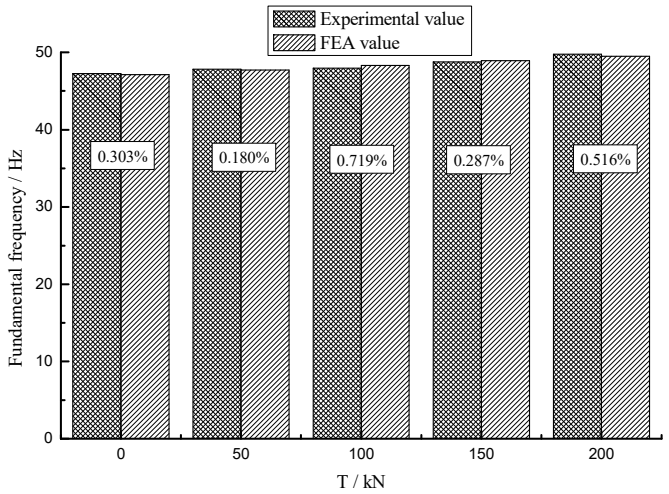

Fig. 8. A comparison of the analyzed result

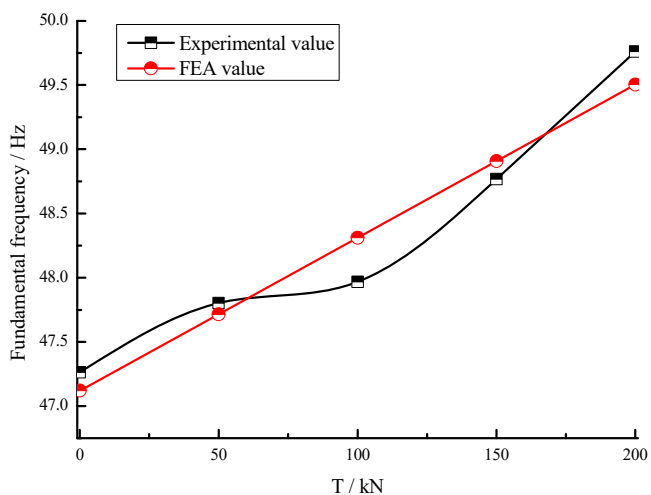

Fig. 9. Curve for the comparison of fundamental frequencies

As shown in the above figure, the fundamental frequency of the beam obtained by adopting the experimental method and FEA method was rather consistent in development trend. However, the measured data of dynamic experiment fluctuated partly. Free from external interference and various accidental factors in the practical experiment, the numerical model presented a relatively stable growth trend. Two methods showed the same growth trend and a small error under various levels of pre-stressed loads, indicating the feasibility and accuracy of two analytical methods.

The fundamental frequency of the beam obtained based on FEA method showed the trend of linear growth with the increase of prestress value, which might be certainly related to the anisotropism of concrete materials in the practical experiment. In the numerical model, the concrete beam defaulted to a homogeneous stress structure. Therefore, the fundamental frequency of the beam showed the trend of linear change.

\subsection{Relationship between eccentric distance, pre-stress and the fundamental frequency}

From the above analysis, it could be found that "hammering method + numerical model + FFT" method proposed by this paper could truly and accurately reflect the dynamic characteristic of the 
pre-stressed concrete beam under different pre-stress values. Thus, the influence of the eccentric distance of pre-stressing tendon on the fundamental frequency of the beam could be studied based on the model. Herein, the numerical model in Fig. 5 was taken as the basic model and the eccentric distance of pre-stressing tendon was considered as a variable to study the change condition of fundamental frequency of the beam when the eccentric distances of pre-stressing tendon were $0 \mathrm{~mm}, 60 \mathrm{~mm}$ and $120 \mathrm{~mm}$ respectively. The schematic diagram for the location of operating conditions at the eccentric distance of pre-stressing tendon was shown in Fig. 10.
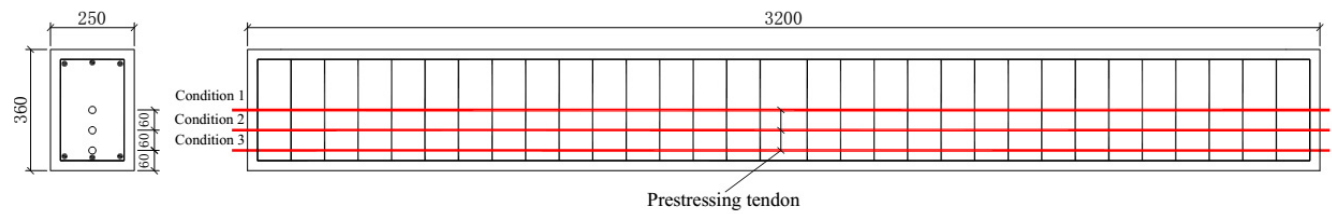

Fig. 10. Operating conditions at the eccentric distance of pre-stressing tendon

Based on the above analytical methods and steps, the fundamental frequency information of the beam under various loading conditions was obtained through applying instantaneous excitation to $1 / 3$ place of the simply supported beam, conducting a dynamic analysis on the beam, extracting the acceleration signals in the midspan of the beam and adopting FFT method. A dynamic analysis on the beam under various operating conditions was conducted to obtain the relationship among the fundamental frequency of the pre-stressed beam, prestress value and eccentric distance according to different three eccentric distances mentioned above when pre-stress values were $0 \mathrm{kN}$, $50 \mathrm{kN}, 100 \mathrm{kN}, 150 \mathrm{kN}$ and $200 \mathrm{kN}$. The specific numerical values were shown as below.

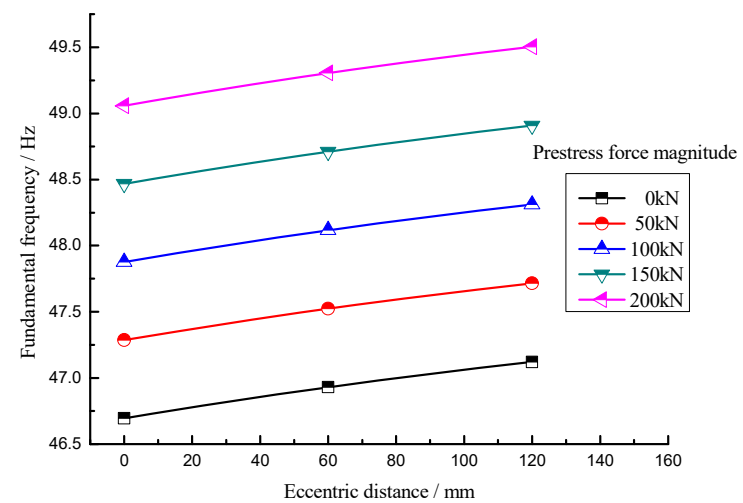

Fig. 11. Relationship between the fundamental frequency of the beam, prestress value and eccentric distance

As shown in the above figure, the fundamental frequency of the pre-stressed beam was increased with the increase of pre-stress value and both of them showed the same growth trend under the operating condition of various eccentric distances. Under the operating condition of the same pre-stress value, the fundamental frequency of the beam with a large eccentric distance was greater than that with a small eccentric distance. Namely the fundamental frequency of the beam was enlarged with the increasing eccentric distance of prestressing tendon. Eccentric distance really had a great influence on the fundamental frequency of the pre-stressed concrete beam.

This phenomenon could be explained through the effective stiffness of the beam. In the case of certain pre-stress value, the enlargement of eccentric distance would increase the flexural stiffness of the beam. Namely, the enlargement of eccentric distance would increase the effective stiffness of the beam so that the fundamental frequency of the beam was increased dramatically. However, the decrease of eccentric distance would lead to the limited increase amplitude of effective stiffness of the beam, which thus resulted in the limited increase amplitude of 
fundamental frequency of the beam. From the perspective of acceptance, the bottom of the pre-stressed concrete beam was in tension when external vertical loads were applied to it. Namely, the position with a large eccentric distance was in tension. Based on plane cross-section assumption, the axial part of the beam was a neutral axis. Therefore, it was the most reasonable to place pre-stressing tendon at the bottom of the beam. The eccentric distance of pre-stressing tendon placed at the bottom was the largest. From the above analysis, it could be noticed that the fundamental frequency of the base would witness great changes. As a result, it was feasible to detect the effective prestress of the pre-stressed concrete beam in actual use based on this theory.

\subsection{Relationship between counterweight, the fundamental frequency and pre-stress}

Considering that countless vehicles will pass the bridge put into operation in reality, it is not realistic to test the fundamental frequency of the beam through cutting off traffic. Test on the fundamental frequency of the beam under normal traffic condition involves the relationship between counterweight, fundamental frequency and pre-stress. To study the relationship among three parts, this paper will apply the concentrated mass of $0 \mathrm{kN}, 300 \mathrm{kN}$ and $600 \mathrm{kN}$ at two $1 / 3$ places and conduct a dynamic analysis on the simply supported beam accordingly after loading each level of pre-stress to the beam. Three kinds of loading conditions were shown in Fig. 12.

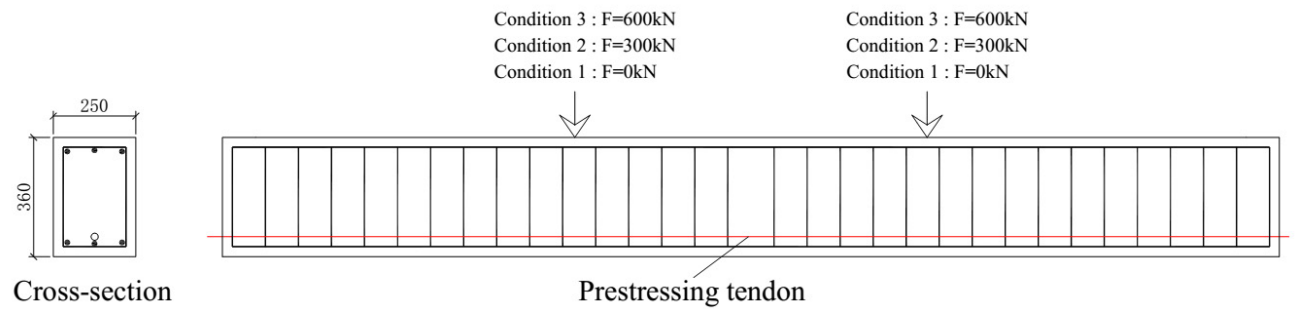

Fig. 12. Loading conditions of counterweight loads

According to the above loading proposal, counterweight was loaded accordingly to the beam. Later, a dynamic analysis on the beam was conducted based on "hammering method + numerical model + FFT" method to obtain the fundamental frequency information of the beam under different pre-stress values and counterweight. The analyzed result of the pre-stressed concrete beam shown in Fig. 13.

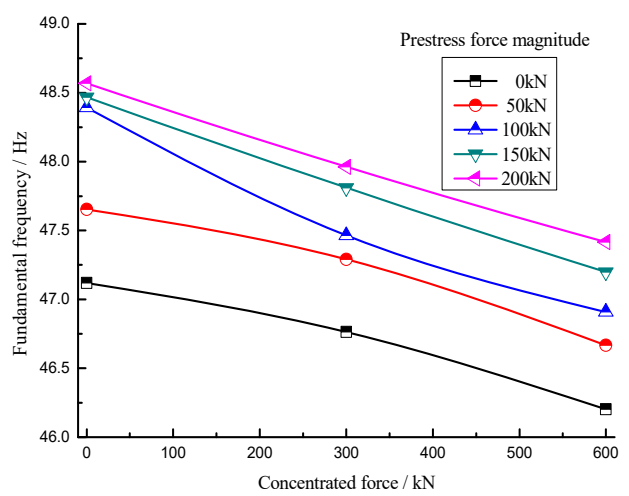

Fig. 13. Relationship between the fundamental frequency, pre-stress and counterweight

As shown in Fig. 13, the fundamental frequency of the beam was decreased with the increase of counterweight at the upper part of the beam in case of certain pre-stress value. Taking the pre-stressed condition of $T=100 \mathrm{kN}$ as an example. After the upper counterweight of the beam 
was increased from $0 \mathrm{kN}$ to $60 \mathrm{kN}$, the fundamental frequency of the beam was decreased from $48.3928 \mathrm{~Hz}$ to $46.9081 \mathrm{~Hz}$, which was decreased by $3.1 \%$. Other levels of loading conditions also showed the same change trend, which proved that the fundamental frequency of the beam and counterweight at the upper part of the beam showed certain mutual influence and regularity. Namely, the fundamental frequency of the beam presented a decreasing trend along with the bob-weight at the upper part of the beam in case other conditions of the fundamental frequency were consistent.

This phenomenon could also be explained through the increased distributed mass of the beam. Namely, the distributed mass of the beam was equivalently increased to some degree due to the existence of concentrated loads. In the process of vibration, the upper loads of the beam did not appear, but move all the time, which would influence the stiffness matrix of the beam in the process of vibration and then the fundamental frequency of the beam. After the above relationship was obtained, it was required to consider redundant bob-weights at the upper part of the beam in the process of detection when effective pre-stress of the actual bridge was detected. In this way, the real dynamic information of the beam could be obtained so as to accurately deduce the health condition of the beam.

\section{Conclusions}

With increasing application of the pre-stress concrete structure in bridges, fan towers, large-span structures, dynamic characteristics of these structures will be especially important as these structures need to bear corresponding static and dynamic environmental loads during operation. At present, researches on fundamental frequency of the pre-stress concrete structures are in continuous exploration. Most of these researches focus on modifications of theoretical formulas and finite element models. There are few researches which combine finite elements with experiments. Meanwhile, researches which combine four factors including fundamental frequency, pre-stress value, balance weight and eccentric distance are fewer. Based on experiments of the pre-stress concrete simply supported beam model and finite element analysis, the paper studies the relations between fundamental frequency, pre-stress value and balance weight, and the eccentric distance. Major conclusions obtained by the paper are as follows:

1) Experiment results obtained in this paper are similar to those by a lot of scholars. Relations between fundamental frequency and pre-stress value of a pre-stress concrete beam do not match with the theory of "Euler-Bernoulli Equation". The theory cannot effectively explain the relations between fundamental frequency and stress value of a pre-stress beam. Its analysis results are contrary to the experimental results, verifying research achievements obtained by the previous scholars;

2) It is found in the fundamental frequency experiment of a pre-stress concrete simply supported beam model that the beam fundamental frequency will increase with the increase of pre-stress value. Both the experiment results and the computational results reflected this tendency.

3) The hammering method could effectively obtain actual dynamic responses of the pre-stress concrete simply supported beam. Through analysis of signals in the beam span, relatively actual beam fundamental frequency information could be obtained. When the hammering method is used in the simulation analysis, the analysis results are similar to experimental results, and errors are small, verifying feasibility and accuracy of the analysis method.

4) The research obtained relations between fundamental frequency, pre-stress value, eccentric distance and balance weight. Specifically, when the pre-stress value is a constant, the beam fundamental frequency will increase with the increase of eccentric distance of the pre-stress rebar. When other conditions are consistent, the beam fundamental frequency will be decreased with the balance weight on the upper part of beams. 


\section{References}

[1] Lee E. T., Eun H. C. Damage detection of beam structure using response data measured by strain gages. Journal of Vibroengineering, Vol. 16, Issue 1, 2014, p. 147-155.

[2] Kim J. T. Damage identification in beam-type structures: frequency-based method vs modeshape-based method. Engineering Structures, Vol. 25, Issue 1, 2003, p. 57-67.

[3] Chan T. H. T., Yung T. H. A theoretical study of force identification using prestressed concrete bridges. Engineering Structures, Vol. 22, Issue 11, 2000, p. 1529-1537.

[4] Fang X. Structural damage detection using neural network with learning rate improvement. Computers and Structures, Vol. 83, Issues 25-26, 2005, p. 2150-2161.

[5] Shi L. N., He H. X., Yan W. M. Prestress force identification for externally prestressed concrete beam based on frequency equation and measured frequencies. Structural and Mechanics, Vol. 2014, 2014.

[6] Pakar I., Bayat M. Analytical study on the non-linear vibration of Euler-Bernoulli beams. Journal of Vibroengineering, Vol. 14, Issue 1, 2012, p. 216-224.

[7] Dai K., Chen S. Vibration of spun-cast prestressed concrete poles. Conference Proceedings of the Society for Experimental Mechanics Series, 2007.

[8] Dall'Asta A., Dezi L. Discussion of prestress force effect on vibration frequency of concrete bridges. Journal of Structural Engineering, Vol. 122, Issue 4, 1996, p. 458-458.

[9] Kerr A. On the dynamic response of a prestressed beam. Journal of Sound and Vibration, Vol. 49, Issue 4, 1976, p. 569-573.

[10] Hamed E., Frostig Y. Natural frequencies of bonded and unbonded prestressed beams - prestress force effects. Journal of Sound and Vibration, Vol. 295, Issue 1, 2006, p. 28-39.

[11] Saiidi M., Douglas B., Feng S. Prestress force effect on vibration frequency of concrete bridges. Mathematical Problems in Engineering, Vol. 120, Issue 7, 1994, p. 2233-2241.

[12] Zhang Y. T., Wang X. L., Li R. G. A study on the theoretical analysis and test of vibration frequency of the full-prestressed beam. Engineering Mechanics, Vol. 24, Issue 8, 2007, p. 116-120.

[13] Zhou W. Y., Li D. X. Experimental research on a vibration isolation platform for momentum wheel assembly. Journal of Sound and Vibration, Vol. 332, Issue 5, 2013, p. 1157-1171.

[14] Mhia Md, Zaglul Shahadat, Takeshi Mizuno Active vibration isolation using negative stiffness and displacement cancellation controls: Comparison based on vibration isolation performance. Control Engineering Practice, Vol. 37, 2015, p. 55-66.

[15] Liu X. Frequency response function shape-based methods for structural damage localisation. Mechanical System and Signal Processing, Vol. 23, Issue 4, 2009, p. 1243-1259.

[16] Lu Z. R., Law S. S. Identification of prestress force from measured structural responses. Mechanical System and Signal Processing, Vol. 20, Issue 8, 2006, p. 2186-2199.

[17] Bandara R. P., Chan T. H. T., Thambiratnam D. P. The three-stage artificial neural network method for damage assessment of building structures. Australian Journal of Structural Engineering, Vol. 14, Issue 1, 2013, p. 13-25.

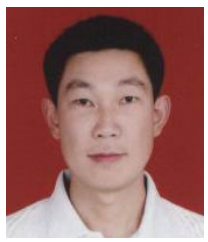

Li Jie received his Master degree in engineering from Changsha University of Science and Technology. Currently, he is a lecturer in the Department of Road and Bridge Engineering of Xinjiang Vocational and Technical College of Communications, which is based in Urumqi, Xinjiang, China. His major research interests include communication and transportation engineering and road and bridge engineering.

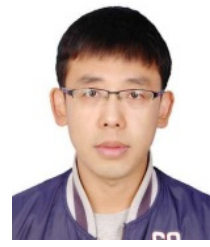

Feng Zhang received his Doctor degree from College of Environmental and Resource Sciences, Zhejiang University. Now he was working at Xinjiang Vocational and Technical College of Communications. His interest includes communication and transportation engineering. 